\title{
Using simulation to assess the impacts of the adoption of a fast-track system for hospital emergency services
}

\author{
Yong-Hong KUO*, Janny M.Y. LEUNG**, Colin A. GRAHAM***, Kelvin K.F. TSOI****,***** and \\ Helen M. MENG****,****** \\ * Department of Industrial and Manufacturing Systems Engineering, The University of Hong Kong \\ Pokfulam Road, Hong Kong \\ E-mail: yhkuo@hku.hk \\ ** Shenzhen Key Laboratory of IoT Intelligent Systems and Wireless Network Technology, The Chinese University of Hong Kong \\ Shenzhen, Guangdong, 518172, P.R. China \\ ${ }^{* * *}$ Accident and Emergency Medicine Academic Unit, The Chinese University of Hong Kong \\ Shatin, New Territories, Hong Kong \\ **** Stanley Ho Big Data Decision Analytics Research Centre, The Chinese University of Hong Kong \\ Shatin, New Territories, Hong Kong \\ ***** School of Public Health and Primary Care, The Chinese University of Hong Kong \\ Shatin, New Territories, Hong Kong \\ ***** Department of Systems Engineering and Engineering Management, The Chinese University of Hong Kong \\ Shatin, New Territories, Hong Kong
}

Received: 24 November 2017; Revised: 24 April 2018; Accepted: 14 May 2018

\begin{abstract}
Overcrowding in hospital emergency departments has been a longstanding global issue. Due to the steadily increasing patient demand, the higher complexity of cases and the limited resources available in hospitals, this overcrowding problem has become more challenging. The overcrowding problem is urgently important as the provision of the necessary medical services to patients may be delayed, leading to various adverse consequences. In order to alleviate overcrowding, hospital administrators have been studying and implementing different solutions for the enhancement of system efficiency. In this paper, we present our work that applies system simulation to model the operations and patient flows at a hospital emergency department. We examine the implications of a fast service track on the efficiency of service provision. This fast-track system, without a doubt, can benefit some groups of patients who can be provided with more responsive medical services but, at the same time, may increase the waiting time of some other groups of patients. With the simulation model, we are able to assess these possible tradeoffs between the waiting times of different types of patients when the fast track is adopted. Our computational experiments suggest that the adoption of a fast-track system can be more beneficial, in terms of the reduction in overall patient waiting time, to emergency departments which have more urgent patients. Our simulation model is not restricted to modeling this fast-track system only, but can also be adopted to examine different policy implications and "what-if" scenarios when managing hospital emergency services.
\end{abstract}

Keywords : Emergency department, Patient flow, Simulation, Fast track, Healthcare management, Scheduling, Operational efficiency

\section{Introduction}

Overcrowding in emergency departments (EDs) is a challenging and critical global issue (e.g., Cowan and Trzeciak, 2005; Derlet et al., 2001; Horwitz et al., 2010; Miró et al., 1999; Shih et al., 1999). While the problem has drawn considerable attention from the hospital's management and the public for years, the overcrowding situation has been observed to be worsening (Herring et al., 2009; Wilper et al., 2008). EDs have the mission of 
providing critical and urgent patients with timely and effective medical treatments. However, there have been various challenges posed to EDs, such as increased complexity and acuity of patients (Derlet and Richards, 2000), and these challenges have further complicated the overcrowding problem. The overcrowding phenomenon implies that EDs do not have sufficient resources to manage the patient demand. As a result, some patients may have to wait for hours to receive the necessary medical treatments. The postponement of the delivery of the required medical treatments to patients can lead to adverse consequences on patients, which can be as serious as unnecessary deaths of critical patients. Although there has already been much effort attempting to tackle the overcrowding problem, the problem remains unsolved in many hospitals because most of the solutions require a significant amount of investments, e.g., expanding hospital capacities and adding medical resources. In general, these solutions are costly such that many of the hospitals, in particular government hospitals, are unable to afford due to their limited financial budgets. For this reason, hospital administrators have been seeking other potential solutions to enhance the ED operational efficiency subject to fixed budgets.

Prioritization of patients for the provision of emergency services has been a standard practice over the world. Patients are treated according to their level of urgency; the more urgent the patient, the higher priority he/she is given. This practice can help achieve the goal that critically ill patients can be given appropriate medical care in a timely fashion. In general, a patient with greater urgency requires a higher level of attention from more medical staff in an ED. Thus, less urgent patients tend to wait for a much longer time. Some hospitals in the world have adopted a "fast-track" system where less urgent patients (who are expected to have fewer and shorter treatments) are allocated to a designated queue for service. The benefit of having such a designated queue is that patients with low complexity can be discharged much earlier so that the ED becomes less crowded. However, the drawback is that the resources (e.g., medical staff) allocated to the more urgent patients are reduced and these patients may not be treated as responsive as in a non-fast track system. The key to the adoption of a "fast-track" system is to strike a balance: shortening the waiting time of less urgent patients while maintaining the required attention and responsiveness to more urgent patients. In this research, we study the impacts of a fast-track system on the enhancement of the ED performance with a simulation approach. We conduct computational experiments with the simulation model that we have previously developed for an ED in Hong Kong to evaluate the impacts of the fast track. The simulation model can help conduct "what-if" analysis and aid policy makers with decision making in the ED. Some of our previous research on this project can be found in Kuo (2014) and Kuo et al. (2015; 2016 a;b).

EDs in Hong Kong are also suffering from the overcrowding problem. The inappropriate uses of the ED by non-urgent patients (Chung, 2000) and the understaffing problem (Rainer, 2013) together have led to the ED overcrowding situation in Hong Kong. As a result, non-urgent patients, who are the majority of the patients and have the lowest priority in receiving medical treatments, may need to experience a very long waiting time of several hours in EDs. The problems of overcrowding and long patient-waiting-times not only worsen patient satisfaction, but also may be barriers to patient access to appropriate and timely medical care. The characteristics of having the majority of patients being non-urgent and their long waiting time make EDs in Hong Kong a unique example to study the impacts of a fast-track system. With an ED as a baseline, we also consider several other ED settings and explore the effects of this fast-track system under these ED environments.

This paper is organized as follows. In the next section, we provide a literature review of the applications of simulation for analyzing and improving ED operations. In section 3, we present our case study and simulation model. In section 4, we conduct a computational study and examine the impacts of a fast-track system. Section 5 concludes our work.

\section{Literature Review}

Simulation has been adopted for aiding decision-making problems in healthcare delivery systems for over half of a century (e.g., Fetter and Thompson, 1965) and the academic literature on its applications for healthcare management is vast. For an overview, we refer the reader to Jun et al. (1999), Fone et al. (2003), Günal and Pidd (2010), Katsaliaki and Mustafee (2011), Paul et al. (2010), Gul and Guneri (2015), Hu et al. (2018).

While there has been research on developing analytictal models for improving decisions in EDs (e.g., Green et al., 2006; Cochran and Roche 2009; Saghafian et al., 2012), our project aims to develop a model that captures highly complicated problem features (e.g., category-dependent and non-stationary patient arrival process, time- 
varying staffing levels, interdependent service stations, non-homogeneous service time distributions and patient re-entrants), resulting in a more detailed model of the actual ED environment. For this reason, simulation is adopted as our tool to conduct our case study. A simulation model also takes advantage of being less sensitive to model parameters (Sinreich and Marmor, 2005) and more appropriate for modeling system where resources are critical (Davies and Davies, 1994). Furthermore, a simulation model enables practitioners to explore different "what-if" scenarios without the need of advanced mathematical knowledge.

There have been various successful applications of simulation for aiding decisions in EDs over the world. Rossetti et al. (1999) developed a simulation model of an ED in Charlottesville, Virginia and used the model to assess the impacts of different doctor work schedules on patient throughput and resource utilization. Yeh and Lin (2007) applied simulation with genetic algorithms to optimize the nurse roster and reduce patient waiting time for an ED in Central Taiwan. Ahmed and Alkhamis (2009) combined simulation and optimization to determine the staffing levels of different types of medical staff (e.g., physicians and nurses) in an ED in Kuwait. Their goal was to optimize, with limited financial budgets, both patient throughput and patient total time in the ED.

Wang et al. (2009) applied a simulation approach to identify bottlenecks in the system of an ED in Lyon and examine resource allocation decisions. Abo-Hamad and Arisha (2013) developed a decision support system with the use of simulation to optimize the ED operations in an adult teaching hospital in North Dublin. They used the tool to determine the number of staff and the physical capacity for improving patient experience. They also examined, with their simulation model, the "zero-tolerance" policy regarding exceeding the national 6-hour boarding time and found that this policy has a great impact on reducing the average length of stay of patients. Kuo et al. (2016b) tackled the challenge of incomplete data for modeling service times and developed a simulation model of an ED in Hong Kong. They applied the simulation model to examine the effects of physician heterogeneity (Kuo et al., 2015) and missing patients (Kuo et al., 2016a) on the ED performance. Guo et al. (2016) also faced the problem where service times could not be directly estimated due to missing data and developed a meta-heuristic approach to tackling the problem. In a later paper, Guo et al. (2017) used the simulation model and optimization to determine the staffing levels in an ED in Hong Kong.

There have also been applications of simulation for analyzing effects of fast-track systems on ED performance. García et al. (1995) conducted a simulation study of the ED of Mercy Hospital in Florida and found that a fast-track lane can reduce the total time in system for patients with low priority in the ED by almost $25 \%$ without negatively affecting other patients with higher priority. Connelly and Bair (2004) used a simulation model of an ED in Davis, California, to assess the effectivenesses of a fast-track triage approach and their proposed acuity ratio triage (ART) approach. They reported that their proposed ART approach could reduce the average treatment times for high-acuity patients; however, the tradeoff was the increase in the average service time for low-acuity patients. Kausha et al. (2015) developed an agent-based simulation model to evaluate a fast-track treatment strategy in an ED in Canada. They found that the strategy can reduce the patient waiting time without affecting the overall ED performance.

The above applications show that simulation is an appropriate and powerful tool for evaluating the effectiveness of a fast-track system in an ED. In this paper, we adopt a simulation model to examine the impacts of a fast-track system on ED operational efficiency. We also conduct a sensitivity analysis and study possible tradeoffs between waiting times of urgent and non-urgent patients when the fast track is adopted.

\section{Case Study}

In this section, we present the ED system and patient flow of our case study and our simulation model. This ED provides 24-hour accident and emergency services. Between 7 am and 11pm, mobile patients and non-mobile patients who need equipment for transfer (i.e., those on a trolley or wheelchair) are treated respectively in two separate divisions (referred to as Walking and Non-walking Divisions). Between 11pm and 7am, the two groups of patients undergo the required treatments in the same division for better utilization of the reduced manpower in respect to the less frequent overnight patient visits. The ED adopts a five-point scale triage system (i.e., five categories) to indicate the urgency of a patient; 1 to 5 respectively represents critical, emergency, urgent, standard and non-urgent. In this ED, categories 3 and 4 are the major group of patients, who represent over $95 \%$ of the total patient visits. Assuming that the numbers of category 1, 2, and 5 patients are negligible, 
the proportions of category 3 and category 4 patients are $30 \%$ and $70 \%$ respectively. The triage number gives the priority of receiving medical treatments. The more urgent, the higher priority the patient has. If patients are in the same category, they are treated on a first-come, first-served basis. Categories 1 and 2 patients are the critically-ill patients who need immediate medical treatments and will be rushed to resuscitation rooms promptly after they are admitted to the ED. For other patients, they first register upon arrival and then are assessed by a triage nurse for assigning a triage category. After triage, they wait until being seen by a medical doctor. After consultation with the doctor, some may need to undergo certain medical diagnostic tests (e.g., $\mathrm{X}$-ray and blood tests) and revisit the physician for a follow-up consultation. After all the required tasks are performed, they are either discharged from the hospital or are required to stay in an in-patient ward for further examinations and treatments.

Based on the patient flow, ED layout and actual data collected, we developed a simulation model of the ED using simulation software ARENA. The main logic of our simulation model to represent the patient flow is depicted in Fig. 1. For more details about the development of our simulation model, input calibration procedure, and model verification and validation, we refer the reader to Kuo et al. (2016b).

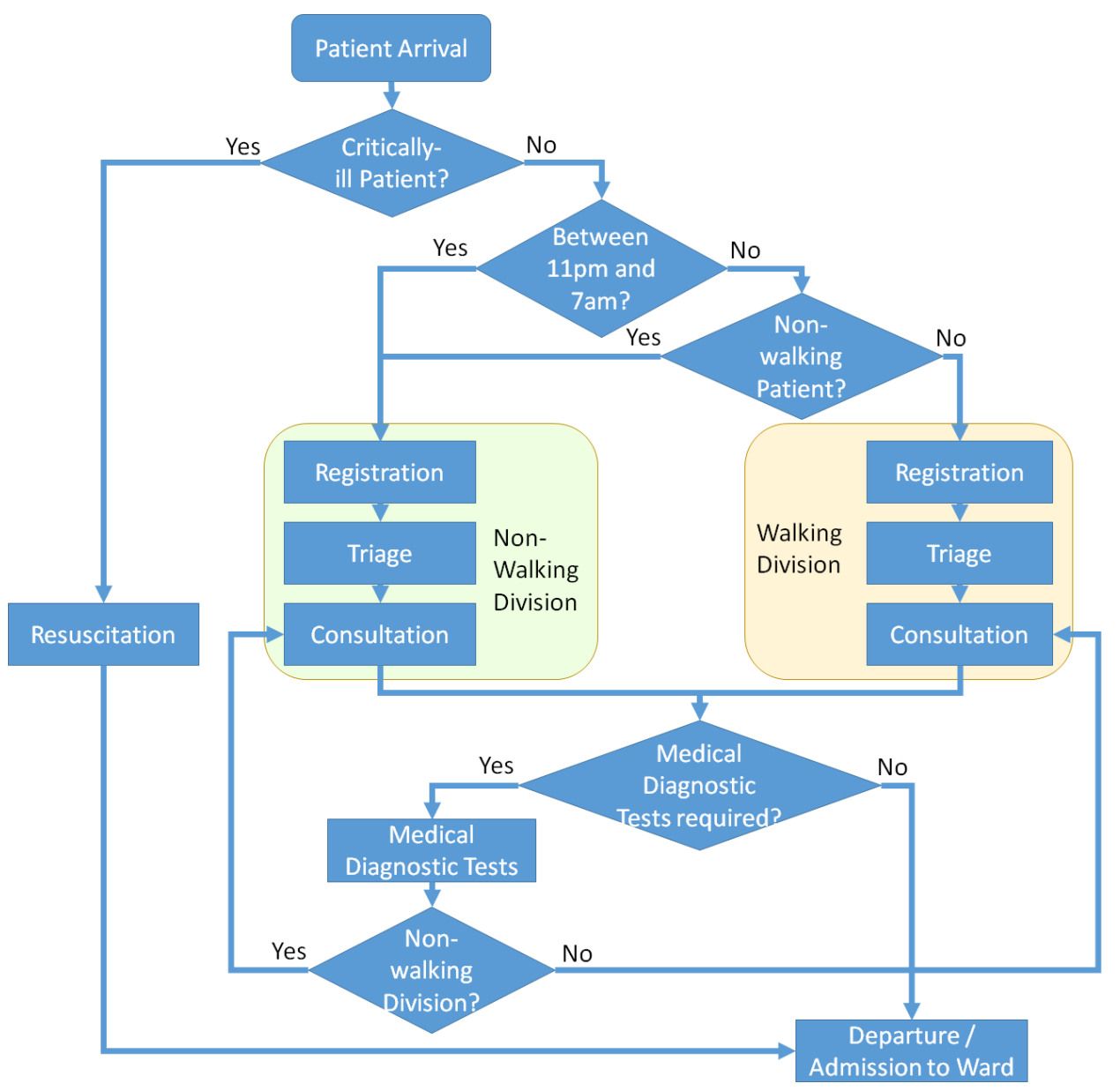

Fig. 1 The main logic of our simulation model of the ED patient flow.

For many EDs, patients are placed in a single queue for consultation, coded in terms of their levels of urgency (i.e., their triage categories). The more urgent patients will be given a higher priority to see a physician. However, these urgent patients are more complex and more likely to spend longer time for physician consultation. As a consequence, those patients with low priority may need to wait for many hours to be seen by a physician. Thus, this leads to the overcrowding problem in the ED (i.e., a large number of patients staying in the ED) and prolonged patient time waiting time. Both result in poor patient experience in the ED. More importantly, the overcrowding environment can also prevent the medical staff from providing effective and efficient services to patients.

The establishments of fast-track systems in EDs aim to facilitate care of the non-urgent cases in a more 
rapid fashion. A fast-track system first streams less complicated patients with low-acuity concerns and then treats them via a dedicated queue, which is different from the regular ones treating more urgent patients with a higher complexity. Fast-track systems have been demonstrated to be practically effective in many EDs (e.g., Considine et al., 2008; Kwa and Blake, 2008; Ieraci et al., 2008) and do not deteriorate the quality of care provided to patients (Sanchez et al. 2006). Although these real applications are shown to be successful, the effect of a fast-track system can vary from one environment to another. The hospital's management may wish to explore its potential benefits and impact on the ED performance before they start to actually implement the system. A simulation approach provides the hospital's management with a powerful way to examine the effect of a fast-track system on the ED operations without jeopardizing the patients.

Given real-world constraints such as limited financial budgets, EDs may not be able to hire an additional physician for this fast-track system. In our experiments, the physician dedicated to this fast track is relocated from the existing staffing resources. This setting enables us to compare the efficiencies of the different systems subject to the same level of resources. We adopt a similar fast-track system used in García et al. (1995), Meislin et al. (1988), and Hampers et al. (1999): a physician is dedicated to the standard and non-urgent patients (i.e., categories 4 and 5 patients in our case study), but these patients can proceed to the regular physicians when the fast-track doctor is occupied and the regular ones are free. The rest of the physicians follow the same practice; patients are seen according to their triage category. In the simulation model, this fast track is established in the process from a triage module to a consultation module in Fig. 1. After a patient is examined by the triage nurse, he/she will be diverted to the regular queue or the fast-track queue according to his/her triage category. Fig. 2 depicts the logic of this fast-track system.

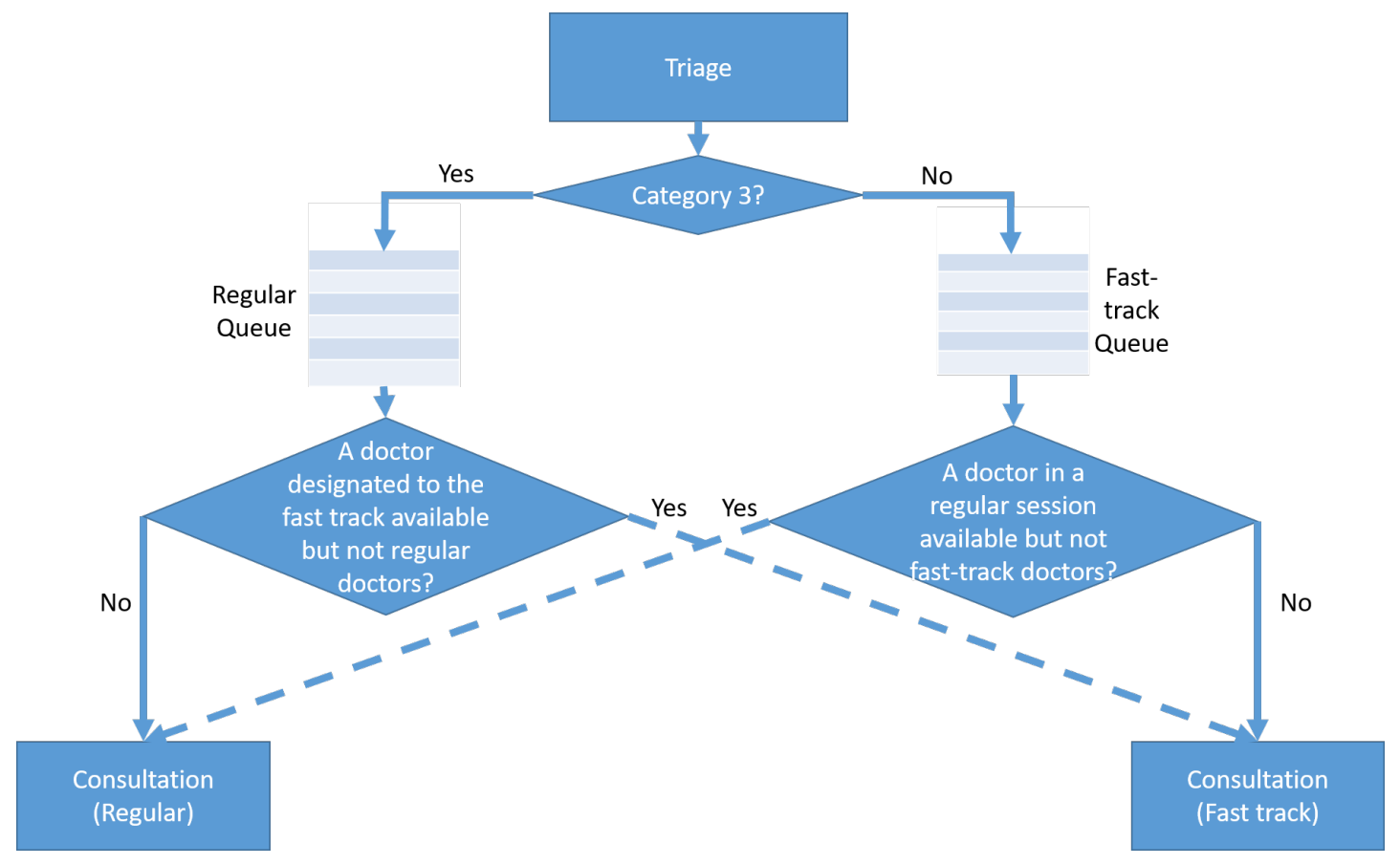

Fig. 2 The logic of the fast-track system.

\section{Simulation Experiments: Examining the Effectiveness of the Fast-Track System}

In this section, we conduct simulation experiments to examine the impacts of a fast-track system on the ED performance.

\subsection{Computational instances}

For the baseline simulation model in our computational study, we use the actual patient arrival rates to the ED, service-time distributions estimated in our previous work (Kuo et al. 2016b), actual staff work schedule as the model input. In our simulation model, all types of patients are generated and they will go through all the procedures as described previously. In order to conduct a more comprehensive study, we also consider other scenarios where the settings in the simulation model vary slightly from the baseline. This aims to conduct a 
sensitivity analysis to ensure that the enhancement of the fast track is robust with respect to the ED environment and to extract insights into the adoption of a fast-track system in general EDs. Table 1 lists the scenarios that we examine in this simulation study.

Scenario S0 is the baseline case, which adopts the current actual settings of the ED. In the following scenarios, all the settings are the same as those of S0, unless stated otherwise. Scenarios S1 and S2 are to examine the effectiveness of the fast track with different proportions of formation of the major patient categories. Scenarios S3 and S4 consider the cases that the number of patient visits to the ED slightly changes by five percent. The fluctuation in the number of patient visits is common for EDs due to the increasing population, epidemics, and seasonal factors. Scenarios S5 to S8 are to examine the effectiveness of the fasttrack system under different scenarios that the required attention paid to patients changes. As there has been reported that ED patients are becoming more complex, the level of required care and the consultation time spent by physicians to patients also vary. For each scenario, we run two sets of simulation experiments, with and without the fast-track system adopted, to examine the impacts of the adoption of the fast-track system.

\subsection{Computational results}

For each problem setting, we ran one hundred replications of 34-day simulation runs, each starting from an empty system (i.e., no patients in the ED initially). In practice, it is very unlikely for an ED to be empty and, therefore, necessary to consider the ED performance in the simulation model after the ED has been providing services to patients for a certain period of time. Thus, the first three days of each replication was a warmup period and excluded from our reported statistics. Our experiments can be interpreted as simulating the patient flows of the ED for one hundred independent months, with the purpose of reducing the effects of the interdependency of samples. In each problem setting, over one million patients were generated (from all these one hundred independent replications). The time stamps of their activities in the simulation were recorded and included for our reported statistics. We measured ED key performance indicators (KPIs) such as the average number of patients in the ED, i.e., work in process (WIP), net time between registration and consultation, i.e., patient waiting time (to first consultation), and doctor utilization. From the simulations, we computed the averages of these KPIs among the one hundred replications, as reported in Table 2. Fig. 3 visualizes these KPIs (using the primary axis scale) and their absolute and percentage changes (using the secondary axis scale) due to the adoption of the fast-track system.

Table 1 Scenarios in our computational study.

\begin{tabular}{cl}
\hline Scenario & Description \\
\hline S0 & The simulation model adopts the original settings. \\
S1 & The proportions of category 3 and category 4 patients are $20 \%$ and $80 \%$ respectively (assuming the numbers of \\
& category 1 and 2 patients are negligible). \\
S2 & The proportions of category 3 and category 4 patients are $40 \%$ and $60 \%$ respectively (assuming the numbers of \\
& category 1 and 2 patients are negligible). \\
S3 & All the patient arrival rates decrease by $5 \%$. \\
S4 & All the patient arrival rates increase by $5 \%$. \\
S5 & The average of the consultation time for category 3 patients decreases by $5 \%$. \\
S6 & The average of the consultation time for category 3 patients increases by $5 \%$. \\
S7 & The average of the consultation time for category 4 patients decreases by $5 \%$. \\
S8 & The average of the consultation time for category 4 patients increases by $5 \%$. \\
\hline
\end{tabular}

In all the scenarios, the average number of patients in the ED and the overall patient waiting time were reduced when the fast track was adopted.

However, as expected, there is a tradeoff between the waiting times of category 3 and category 4 patients; after the fast-track system was implemented, on average, category 4 patients could be seen by a physician earlier but category 3 patients had to wait for a longer time for consultation because one of the regular physicians was reallocated to the fast track. As the majority of patients in the ED are in category 4 and their absolute reductions in waiting time are significantly higher than those of category 3 patients, the overall waiting time could thus be reduced. The hospital administrators will have to determine whether this increase in waiting time of category 3 patients is acceptable. For example, in the current environment of the ED (i.e., scenario S0), although the adoption of the fast track increased the waiting time of category 3 patients by a large percentage $(18.17 \%)$, the absolute increase was only around 4 minutes. If this is acceptable, the hospital administrators 
Kuo, Leung, Graham, Tsoi and Meng,

Journal of Advanced Mechanical Design, Systems, and Manufacturing, Vol.12, No.3 (2018)
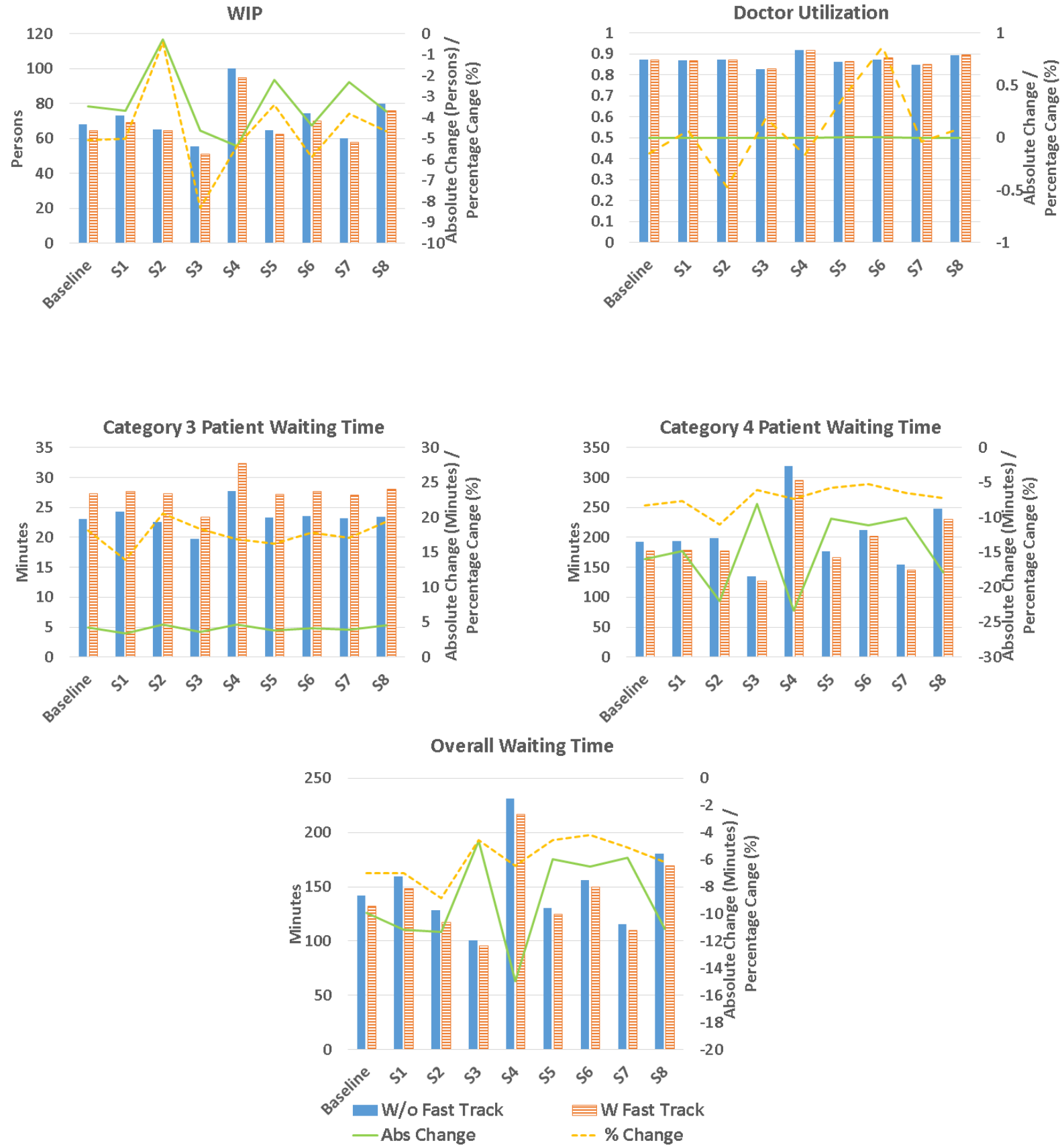

Fig. 3 Key performance metrics resulting from the ED systems with and without fast track adopted under difference the scenarios. 
Kuo, Leung, Graham, Tsoi and Meng,

Journal of Advanced Mechanical Design, Systems, and Manufacturing, Vol.12, No.3 (2018)

Table 2 Key performance metrics resulting from the ED systems with and without fast track adopted under difference the scenarios.

\begin{tabular}{|c|c|c|c|c|c|c|}
\hline \multirow[b]{2}{*}{ Scenario } & \multirow{2}{*}{$\begin{array}{c}\text { Is fast } \\
\text { track } \\
\text { adopted? }\end{array}$} & \multirow[b]{2}{*}{ WIP } & \multicolumn{3}{|c|}{ Waiting time (minutes) } & \multirow{2}{*}{$\begin{array}{c}\text { Doctor } \\
\text { utilization }\end{array}$} \\
\hline & & & Category 3 & Category 4 & Overall & \\
\hline \multirow[t]{2}{*}{ S0 } & No & 68.2 & 23.08 & 192.99 & 142.02 & 0.87 \\
\hline & Yes & 64.74 & 27.28 & 177.05 & 132.12 & 0.8707 \\
\hline \multirow{2}{*}{$\begin{array}{c}\text { Absolute difference } \\
\% \text { change }\end{array}$} & $\mathrm{N} / \mathrm{A}$ & -3.46 & 4.19 & -15.93 & -9.89 & -0.0013 \\
\hline & $\mathrm{N} / \mathrm{A}$ & $-5.08 \%$ & $18.17 \%$ & $-8.26 \%$ & $-6.97 \%$ & $-0.15 \%$ \\
\hline \multirow[t]{2}{*}{ S1 } & No & 73.03 & 24.33 & 193.58 & 159.73 & 0.8681 \\
\hline & Yes & 69.37 & 27.7 & 178.75 & 148.54 & 0.8688 \\
\hline \multirow{2}{*}{$\begin{array}{c}\text { Absolute difference } \\
\% \text { change }\end{array}$} & $\mathrm{N} / \mathrm{A}$ & -3.65 & 3.37 & -14.82 & -11.18 & 0.0006 \\
\hline & $\mathrm{N} / \mathrm{A}$ & $-5.01 \%$ & $13.88 \%$ & $-7.66 \%$ & $-7 \%$ & $0.07 \%$ \\
\hline \multirow[t]{2}{*}{$\mathrm{S} 2$} & No & 65 & 22.62 & 199.06 & 128.48 & 0.8748 \\
\hline & Yes & 64.74 & 27.28 & 177.05 & 117.14 & 0.8707 \\
\hline \multirow{2}{*}{$\begin{array}{c}\text { Absolute difference } \\
\% \text { change }\end{array}$} & $\mathrm{N} / \mathrm{A}$ & -0.26 & 4.65 & -22 & -11.33 & -0.0041 \\
\hline & $\mathrm{N} / \mathrm{A}$ & $-0.41 \%$ & $20.6 \%$ & $-11.05 \%$ & $-8.82 \%$ & $-0.47 \%$ \\
\hline \multirow[t]{2}{*}{ S3 } & No & 55.69 & 19.75 & 134.81 & 100.29 & 0.8285 \\
\hline & Yes & 51.07 & 23.38 & 126.69 & 95.7 & 0.8301 \\
\hline \multirow{2}{*}{$\begin{array}{c}\text { Absolute difference } \\
\% \text { change }\end{array}$} & $\mathrm{N} / \mathrm{A}$ & -4.62 & 3.62 & -8.11 & -4.59 & 0.0016 \\
\hline & $\mathrm{N} / \mathrm{A}$ & $-8.3 \%$ & $18.35 \%$ & $-6.02 \%$ & $-4.58 \%$ & $0.19 \%$ \\
\hline \multirow[t]{2}{*}{$\mathrm{S} 4$} & No & 100.18 & 27.7 & 318.82 & 231.49 & 0.9177 \\
\hline & Yes & 94.82 & 32.36 & 295.47 & 216.54 & 0.9163 \\
\hline \multirow{2}{*}{$\begin{array}{c}\text { Absolute difference } \\
\% \text { change }\end{array}$} & $\mathrm{N} / \mathrm{A}$ & -5.35 & 4.66 & -23.35 & -14.94 & -0.0014 \\
\hline & $\mathrm{N} / \mathrm{A}$ & $-5.35 \%$ & $16.82 \%$ & $-7.32 \%$ & $-6.46 \%$ & $-0.16 \%$ \\
\hline \multirow[t]{2}{*}{ S5 } & No & 64.62 & 23.35 & 176.44 & 130.51 & 0.8613 \\
\hline & Yes & 62.42 & 27.15 & 166.26 & 124.53 & 0.8647 \\
\hline \multirow{2}{*}{$\begin{array}{c}\text { Absolute difference } \\
\% \text { change }\end{array}$} & $\mathrm{N} / \mathrm{A}$ & -2.19 & 3.79 & -10.17 & -5.98 & 0.0034 \\
\hline & $\mathrm{N} / \mathrm{A}$ & $-3.4 \%$ & $16.26 \%$ & $-5.77 \%$ & $-4.58 \%$ & $0.39 \%$ \\
\hline \multirow[t]{2}{*}{$\mathrm{S} 6$} & No & 74.47 & 23.5 & 212.63 & 155.89 & 0.8744 \\
\hline & Yes & 70.08 & 27.67 & 201.52 & 149.37 & 0.882 \\
\hline \multirow{2}{*}{$\begin{array}{c}\text { Absolute difference } \\
\% \text { change }\end{array}$} & $\mathrm{N} / \mathrm{A}$ & -4.39 & 4.17 & -11.1 & -6.52 & 0.0076 \\
\hline & $\mathrm{N} / \mathrm{A}$ & $-5.9 \%$ & $17.77 \%$ & $-5.22 \%$ & $-4.18 \%$ & $0.87 \%$ \\
\hline \multirow[t]{2}{*}{ S7 } & No & 60.05 & 23.13 & 154.81 & 115.31 & 0.8489 \\
\hline & Yes & 57.75 & 27.07 & 144.74 & 109.44 & 0.8486 \\
\hline \multirow{2}{*}{$\begin{array}{c}\text { Absolute difference } \\
\% \text { change }\end{array}$} & $\mathrm{N} / \mathrm{A}$ & -2.3 & 3.94 & -10.07 & -5.87 & -0.0003 \\
\hline & $\mathrm{N} / \mathrm{A}$ & $-3.83 \%$ & $17.04 \%$ & $-6.51 \%$ & $-5.09 \%$ & $-0.04 \%$ \\
\hline \multirow[t]{2}{*}{ S8 } & No & 79.79 & 23.46 & 247.61 & 180.36 & 0.8937 \\
\hline & Yes & 76.09 & 28 & 229.79 & 169.26 & 0.8945 \\
\hline \multirow{2}{*}{$\begin{array}{c}\text { Absolute difference } \\
\% \text { change }\end{array}$} & $\mathrm{N} / \mathrm{A}$ & -3.7 & 4.54 & -17.81 & -11.1 & 0.0008 \\
\hline & $\mathrm{N} / \mathrm{A}$ & $-4.64 \%$ & $19.37 \%$ & $-7.19 \%$ & $-6.16 \%$ & $0.09 \%$ \\
\hline
\end{tabular}


may consider the adoption of a fast track because the majority of patients (category 4 patients) can benefit from the fast-track system but, at the same time, the waiting time of category 3 patients is still within the acceptable range.

From the simulation results, we have the following observations.

(1) The waiting time of category 4 patients is quite sensitive to the number of attendances and the consultation durations. A small change (5\%) in the arrival rates or the average of the consultation time can lead to a big increase in the waiting time of category 4 patients (by comparing S3 - S8 with S0). This implies that the current ED environment is overloaded.

( 2 ) There is no significant change in doctor utilization due to the adoption of the fast-track system under all scenarios. This indicates that the fast-track system does not increase or reduce the physician workloads.

( 3 ) The reduction in the overall patient waiting time due to the adoption of the fast-track system is larger when there are more category 3 patients (a reduction of $-8.82 \%$ in overall patient waiting time in S3, which is the largest among all scenarios). The reason is that when there are more category 3 patients, category 4 patients are expected to wait for a longer time. The fast track allows category 4 patients to bypass this large group of category 3 patients and, therefore, reduces their waiting time more significantly. This suggests that the adoption of a fast track can be more beneficial to EDs that have more patients of higher levels of medical urgency. However, when a fast-track system is adopted, the hospital's management needs to examine if the increase in the waiting time of the urgent patients is acceptable and to ensure that the care provided to those urgent patients is adequate.

\section{Conclusions}

Overcrowding in EDs is one of the most challenging issues for hospital's management. Practitioners and researchers have been trying different ideas to tackle this challenge for many years but this problem remains unsolved in many EDs. In this paper, we adopt a simulation approach and conduct computational experiments to examine potential effect of a fast-track system on the ED performance. This fast-track is dedicated to standard and non-urgent patients, who are the patients suffering from the prolonged waiting time for physician consultation. We found that the adoption of the fast-track system may alleviate the ED overcrowding situation by reducing the number of patients in the ED and the overall patient waiting time. We also observed that there is a tradeoff between the waiting times of urgent patients and non-urgent patients when a fast-track system is adopted. The hospital administrators will have to determine if the increased waiting time of the urgent patients is acceptable in return for the reduction in the waiting time of the non-urgent patients. A simulation model enables the policy maker to foresee the benefit from the adoption of a fast-track system and its detriment. From the computational experiments, we also found that a fast-track system can achieve a larger reduction in the overall patient waiting time for EDs which have more patients of higher levels of medical urgency. This paper demonstrates the use of simulation modeling for forecasting the outcomes of possible changes in the system. Our simulation model is not restricted only to the examination of the impacts of a fast-track system, but can also be adopted for other assessments of potential solutions to improving ED operations (e.g., evaluating the effects of different staff schedules, forecasting the ED performance resulting from the growing patient demand, and examining the benefits of increasing the ED capacity).

\section{Acknowledgement}

This research is supported by Health and Medical Research Fund, Food and Health Bureau, the Hong Kong SAR Government [Project No: 14151771], General Research Fund, the Research Grants Council of Hong Kong [Project No.: 14202115], and Shenzhen Science and Technology Innovation Committee [Grant No.:

ZDSYS20170725140921348]. The authors would like to thank Mr. Stones Wong, Operations Manager of the Emergency Department of the Prince of Wales Hospital in Hong Kong, for his assistance in collecting data.

\section{References}

Abo-Hamad, W. and Arisha, A., Simulation-based framework to improve patient experience in an emergency department, European Journal of Operation Research, Vol.224, No.1 (2013), pp. 154-166. 
Ahmed, M.A. and Alkhamis, T.A., Simulation optimization for an emergency department healthcare unit in Kuwait, European Journal of Operational Research, Vol.198, No.3, (2009), pp. 936-942.

Chung, C.H., Emergency department misuse and administrative interventions, Hong Kong Journal of Emergency Medicine, Vol.7, No.4 (2000), pp. 220-229.

Cochran, J.K., and Roche, K.T., A multi-class queuing network analysis methodology for improving hospital emergency department performance, Computers \& Operations Research, Vol.36, No.5 (2009), pp. 14971512 .

Connelly, L.G. and Bair, A.E., Discrete event simulation of emergency department activity: a platform for system-level operations research, Academic Emergency Medicine, Vol.11, No.11 (2004), pp. 1177-1185.

Considine, J., Kropman, M., Kelly, E. and Winter, C., Effect of emergency department fast track on emergency department length of stay: a case-control study, Emergency Medicine Journal, Vol.25, No.12 (2008), pp. $815-819$.

Cowan, R.M., and Trzeciak, S., Clinical review: emergency department overcrowding and the potential impact on the critically ill, Critical Care, Vol.9, No.3 (2005), pp. 291-295.

Derlet, R.W. and Richards, J.R., Overcrowding in the nation's emergency departments: Complex causes and disturbing effects, Annals of Emergency Medicine, Vol.35, No.1 (2000), pp. 63-68.

Derlet, R.W., Richards, J.R. and Kravitz, R.L., Frequent overcrowding in U.S. emergency departments, Academic Emergency Medicine, Vol.8, No.2 (2001), pp. 151-155.

Fetter, R.B. and Thompson, J.D., The simulation of hospital systems, Operations Research, Vol.13, No.5 (1965), pp. 689-711.

Fone, D., Hollinghurst, S., Temple, M., Round, A., Lester, N., Weightman, A., Roberts, K., Coyle, E., Bevan, G. and Palmer, S., Systematic review of the use and value of computer simulation modelling in population health and health care delivery, Journal of Public Health Medicine, Vol.25, No.4 (1965), pp. 325-335.

FShih, F.Y., Ma, H.M., Chen, S.C., Wang, H.P., Fang, C.C., Shyu, R.S., Huang, G.T. and Wang, S.M., ED overcrowding in Taiwan: Facts and strategies, The American Journal of Emergency Medicine, Vol.17, No.2 (1999), pp. 198-202.

García, M.L., Centeno, M., Rivera, C. and DeCario, N., Reducing time in an emergency room via a fast-track, Proceedings of the 1995 Winter Simulation Conference (1995), pp. 1048-1053.

Green, L.V., Soares, J., Giglio, J.F. and Green, R.A., Using queuing theory to increase the effectiveness of emergency department provider staffing, Academic Emergency Medicine, Vol.13, No.1 (2006), pp. 61-68.

Gul, M. and Guneri, A.F., A comprehensive review of emergency department simulation applications for normal and disaster conditions, Computers \& Industrial Engineering, Vol.83 (2015), pp. 327-344.

Günal, M.M. and Pidd, M., Discrete event simulation for performance modelling in health care: a review of the literature, Journal of Simulation, Vol.4, No.1 (2010), pp. 42-51.

Guo, H., Gao, S., Tsui, K. L., Niu, T., Simulation optimization for medical staff configuration at emergency department in Hong Kong. IEEE Transactions on Automation Science and Engineering, Vol.14, No.4 (2017), pp. 1655-1665.

Guo, H., Goldsman, D., Tsui, K. L., Zhou, Y., and Wong, S. Y., Using simulation and optimisation to characterise durations of emergency department service times with incomplete data. International Journal of Production Research, Vol.54, No.21(2016), pp. 6494-6511.

Hampers, L.C., Cha, S., Gutglass, D.J., Binns, H.J. and Krug, S.E., Fast track and the pediatric emergency department: resource utilization and patient outcomes, Academic Emergency Medicine, Vol.6, No.11 (1999), pp. 1153-1159.

Herring, A., Wilper, A., Himmelstein, D.U., Woolhandler, S., Espinola, J.A., Brown, D.F. and Camargo, Jr. C.A., Increasing length of stay among adult Visits to U.S. emergency departments, 2001-2005, Academic Emergency Medicine, Vol.16, No.7 (2009), pp. 609-616.

Hu, X., Barnes, S., and Golden, B., Applying queueing theory to the study of emergency department operations: a survey and a discussion of comparable simulation studies. International Transactions in Operational Research, Vol.25, No.1 (2018), pp. 7-49.

Horwitz, L.I., Green, J. and Bradley, E.H., US emergency department performance on wait time and length of visit, Annals of Emergency Medicine, Vol.55, No.2 (2010), pp. 133-141.

Ieraci, S., Digiusto, E., Sonntag, P., Dann, L. and Fox, D., Streaming by case complexity: evaluation of a model 
for emergency department fast track, Emergency Medicine Australasia, Vol.20, No.3 (2008), pp. $241-249$.

Jun, J.B., Jacobson, S.H., and Swisher, J.R., Application of discrete-event simulation in health care clinics: a survey, The Journal of the Operational Research Society, Vol.50, No.2 (1999), pp. 109-123.

Katsaliaki, K. and Mustafee, N. Applications of simulation within the healthcare context, Journal of the Operational Research Society, Vol.62, No.8 (2011), pp. 1431-1451.

Kaushal, A., Zhao, Y., Peng, Q., Strome, T., Weldon, E., Zhang, M. and Chochinov, A., Evaluation of fast track strategies using agent-based simulation modeling to reduce waiting time in a hospital emergency department, Socio-Economic Planning Sciences, Vol.50 (2015), pp. 18-31.

Kuo, Y.H., Integrating simulation with simulated annealing for scheduling physicians in an understaffed emergency department, HKIE Transactions, Vol.21, No.4 (2014), pp. 253-261.

Kuo, Y.H., Leung, J.M.Y. and Graham, C.A., Using simulation to examine the effect of physician heterogeneity on the operational efficiency of an overcrowded hospital emergency department, Journal of Physics: Conference Series, Vol.616, No.1 (2015), Paper No. 012017.

Kuo, Y. H., Leung, J. M.Y. and Graham, C. A., How do missing patients aggravate emergency department overcrowding? a real case and a simulation study, Health Care Systems Engineering for Scientists and Practitioners, Springer Proceedings in Mathematics \& Statistics, Vol. 169 (2016a), pp. 167-177.

Kuo, Y.H., Rado, O., Lupia, B., Leung, J.M.Y. and Graham, C.A., Improving the efficiency of a hospital emergency department: a simulation study with indirectly imputed service-time distributions, Flexible Services and Manufacturing Journal, Vol. 28, No. 1 (2016b), pp. 120-147.

Kwa, P. and Blake, D., Fast track: Has it changed patient care in the emergency department? Emergency Medicine Australasia, Vol.20, No. 1 (2008), pp. 10-15.

Meislin, H.W., Coates, S.A., Cyr, J. and Valenzuela, T., Fast track: urgent care within a teaching hospital emergency department: can it work? Annals of Emergency Medicine, Vol.17, No.5 (1988), pp. $453-456$.

Mielczarek, B. and Uziałko-Mydlikowska, J., Application of computer simulation modeling in the health care sector: a survey, Simulation, Vol.88, No.2 (2012), pp. 197-216.

Miró, O., Antonio, M.T., Jiménez, S., De Dios, A., Sánchez, A., Borrás, A. and Millá J., Decreased health care quality associated with emergency department overcrowding, European Journal of Emergency Medicine, Vol.6, No.2 (1999), pp. 105-107.

Paul, S.A., Reddy, M.C., and DeFlitch, C.J. A systematic review of simulation studies investigating emergency department overcrowding, Simulation, Vol.86, No.8-9 (2010), pp. 559-571.

Rainer, T., Why are Hong Kong's emergency departments so understaffed, Emergency Physicians International, Vol.11 (2013), pp. 26-27.

Rossetti, M.D., Trzcinski, G.F. and Syverud, S.A., Emergency department simulation and determination of optimal attending physician staffing schedules, Proceedings of the 1995 Winter Simulation Conference (1999), pp. 1532-1540.

Saghafian, S., Hopp, W.J., Van Oyen, M.P., Desmond, J.S. and Kronick, S.L., Patient streaming as a mechanism for improving responsiveness in emergency departments, Operations Research, Vol.60, No.5 (2012), pp. 1080-1097.

Sanchez, M., Smally, A.J., Grant, R.J. and Jacobs, L.M., Effects of a fast-track area on emergency department performance, The Journal of Emergency Medicine, Vol.31, No.1 (2006), pp. 117-120.

Wang, T., Guinet, A., Belaidi, A. and Besombes, B., Modelling and simulation of emergency services with ARIS and Arena. case study: the emergency department of Saint Joseph and Saint Luc Hospital, Production Planning and Control, Vol.20, No.6 (2009), pp. 484-495.

Welch, S.J., Asplin, B.R., Stone-Griffith, S., Davidson, S.J., Augustine, J. and Schuur, J., Emergency department operational metrics, measures and definitions: results of the second performance measures and benchmarking summit, Annals of Emergency Medicine, Vol.58, No.1 (2011), pp. 33-40.

Wilper, A.P., Woolhandler, S., Lasser, K.E., McCormick, D., Cutrona, S.L., Bor, D.H. and Himmelstein, D.U., Waits to See an emergency department physician: U.S. trends and predictors, 1997-2004, Health Affairs, Vol.27, No.2 (2008), pp. 84-95.

Yeh J.Y. and Lin, W.S., Using simulation technique and genetic algorithm to improve the quality care of a hospital emergency department, Expert Systems with Applications, Vol.32, No.4 (2007), pp. 1073-1083. 